\title{
Quality Improvement Toward Decreasing High-Risk Medications for Older Veteran Outpatients
}

\author{
Alan J. Zillich, PharmD, ${ }^{a b}$ Kenneth Shay, DDS, MS, ${ }^{c}$ Barbara Hyduke, MSA, ${ }^{d}$ \\ Thomas R. Emmendorfer, PharmD, ${ }^{e}$ Alan M. Mellow, MD, PhD, ${ }^{\text {fg }}$ Steven R. Counsell, MD, ${ }^{\text {hi }}$ \\ Mark A. Supiano, MD, ${ }^{j k}$ Peter Woodbridge, MD, MBA, almn and Pamela Reeves, ${ }^{\circ} D^{o p}$
}

OBJECTIVES: To examine the effectiveness of a quality improvement program to decrease prescribing of high-risk medications.

DESIGN: Single cohort, pre- and postintervention.

SETTING: Regional network of Department of Veterans Affairs medical facilities.

PARTICIPANTS: Outpatient veterans aged 65 and older who received one or more high-risk medications and the prescribing clinicians.

INTERVENTION: A two-stage intervention was implemented. First, a real-time warning message to prescribers appeared whenever one of the high-risk drugs was ordered; second, a personally addressed letter from the Chief Medical Officer asking prescribers to consider discontinuing the high-risk medication along with a copy of the Beers criteria article, a list of suggested alternatives to high-risk medications, and a list of older patients receiving the high-risk medications who had upcoming appointments with these prescribers.

MEASUREMENTS: The primary outcome was the absence of prescribed high-risk medications for all patients in the cohort during the postintervention period. For a subgroup of the cohort whose prescribers received the second-

From the ${ }^{\mathrm{a}}$ Center for Excellence in Implementing Evidence-Based Practices and ${ }^{\mathrm{I}}$ Quality and Clinical Informatics Roudebush Veterans Affairs Medical Center, Indianapolis, Indiana; 'bchool of Pharmacy, Purdue University, West Lafayette, Indiana; ' Geriatric Programs, Office of Geriatrics and Extended Care and ${ }^{\mathrm{d}}$ Geriatrics and Extended Care Service Line and ${ }^{\mathrm{f}}$ Mental Health Service Line, ${ }^{\circ}$ Veterans Healthcare Network 11, Department of Veterans Affairs, Ann Arbor, Michigan; ${ }^{\mathrm{e}}$ Pharmacy Benefits Management, Strategic Health Care Group, Department of Veterans Affairs, Battle Creek, Michigan; ${ }^{\mathrm{g}}$ Section of Geriatric Psychiatry and ${ }^{\mathrm{P}}$ Department of Internal Medicine, University of Michigan, Ann Arbor, Michigan; ${ }^{\mathrm{h}}$ Center for Aging Research and Departments of ${ }^{\mathrm{i}}$ Medicine, ${ }^{\mathrm{m}}$ Pathology and Laboratory Medicine, and ${ }^{\mathrm{n}}$ General Internal Medicine, School of Medicine, Indiana University, Indianapolis, Indiana; ${ }^{\mathrm{j} G e r i a t r i c s ~ R e s e a r c h, ~ E d u c a t i o n ~ a n d ~ C l i n i c a l ~ C e n t e r, ~ V e t-~}$ erans Affairs Salt Lake City, Salt Lake City, Utah; and ${ }^{\mathrm{k}}$ Division of Geriatrics, School of Medicine, University of Utah, Salt Lake City, Utah.

Address correspondence to Alan J. Zillich, Purdue University, Pharmacy Programs-Indianapolis, W7555, Myers Building, 1001 West 10th Street, Indianapolis, IN 46202. E-mail: azillich@purdue.edu

DOI: 10.1111/j.1532-5415.2008.01772.x stage intervention, an additional outcome was the absence of prescribed high-risk medications within the subgroup.

RESULTS: Two thousand seven hundred fifty-three unique patients were identified in the cohort; $1,396(50.7 \%)$ had high-risk medications discontinued, resulting in a significant decrease in the number of patients prescribed high-risk medications from the preintervention period to the postintervention period $(P<.001)$. Of the 801 patients in the subgroup, $72.0 \%(\mathrm{n}=577)$ had high-risk medications discontinued $(P<.001)$.

CONCLUSION: This multimethod intervention significantly decreased prescribing of high-risk medications to older patients. Further studies are needed to confirm the findings. J Am Geriatr Soc 56:1299-1305, 2008.

Key words: Beers criteria; medication prescribing; geriatrics; medication safety

W ith the aging of the population and the introduction of more-complex healthcare technologies, the medical care of elderly people now demands significant effort and resources in health systems throughout the world. One area of increasing concern is the potential toxicities of pharmacological agents used to treat a variety of disorders. In addition to the known side effects of appropriately prescribed drugs is the problem of prescribed agents that, because of age-related toxicities, are inappropriate for use in an older population and are often responsible for significant morbidity and mortality and higher healthcare costs. The Institute of Medicine has suggested that workable solutions must be found to improve unsafe medication practices, decrease drug-related problems, and improve patient safety. ${ }^{1}$

Adverse drug events have been linked to preventable problems in elderly patients such as depression, constipation, falls, immobility, delirium, confusion, and hip fractures. In community-dwelling elderly people, there is a high prevalence of inappropriate medication use. Several criteria 
and indices have been used to quantify the extent of inappropriate use, and reports have observed rates of $20 \%$ to $30 \%{ }^{2-8}$ The widely cited Beers criteria were developed from expert consensus in 1991 for just such a purpose and were updated in 1997 and 2003. ${ }^{9-11}$ The Beers criteria have been applied to develop an evidence-based list of medications that disproportionably place elderly patients at highrisk for adverse drug events in light of the availability of safer alternatives. Within the Veterans Affairs (VA) health system, a recent examination of medication databases revealed that $23 \%$ to $33 \%$ of all veterans aged 65 and older had received a potentially inappropriate medication during fiscal year 2000, as judged according to the Beers criteria. ${ }^{12}$

The key to successful use of criteria such as the Beers criteria for improving medical care involves changing prescribing behaviors. Modification of prescribing has been achieved with variable success in many settings, using techniques including formulary restrictions, collaborative care, academic detailing, broad educational efforts, and computerized alerts. ${ }^{13-22}$ Recent studies demonstrated that implementation of computerized alerts could reduce prescriptions for potentially inappropriate medications to elderly patients. ${ }^{17,18}$ In the current study, a multimethod quality improvement intervention, using a combination of computerized alerts and personalized messages, was used to change prescribing of selected high-risk medications within a region of the VA health system.

\section{METHODS}

\section{Study Setting}

The Veterans Health Administration (VHA), within the U.S. Department of Veterans Affairs, is the largest integrated healthcare system in the country. It is organized into 21 regional networks (called Veterans Integrated Service Networks (VISNs)) and provides a unique opportunity for studying and implementing provider behavior change. Providers are employees of the health system, allowing for close administrative oversight, and the VA's information technology systems allow access to aggregate and provider-specific medical records, scheduling, and pharmacy data. VISN 11 is the VA regional network that consists of eight hospitals and 21 freestanding, community-based outpatient clinics in lower Michigan, most of Indiana, central regions of Illinois, and northeastern Ohio. As part of its ongoing efforts at quality improvement, VISN 11 undertook a network-wide, multimethod intervention aimed at reducing prescriptions for older veterans of five medications deemed "high risk" according to the Beers criteria.

\section{Study Design}

A pre- and postintervention design was used to evaluate the effectiveness of a two-stage intervention aimed at reducing prescription of five high-risk medications. The study received exemption status from the VA institutional review board.

\section{Study Population}

Although prescribers were the target of the two-stage intervention (described below), a patient cohort was identified to examine prescription records related to five high-risk

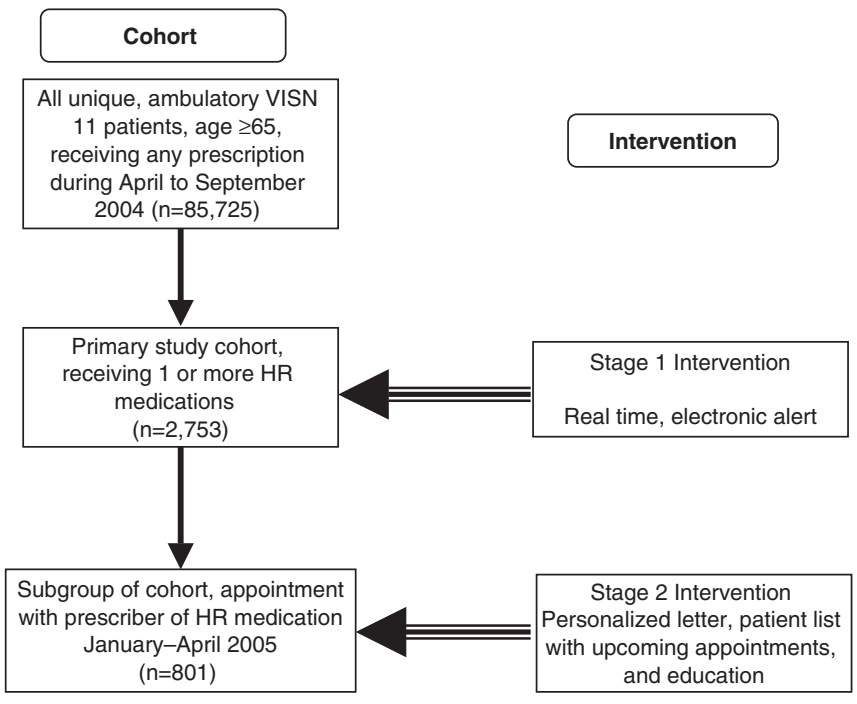

Figure 1. Cohort identification and staged intervention. VISN $=$ Veterans Integrated Service Network. HR = high-risk medications (amitriptyline, chlordiazepoxide, diazepam, doxepin, imipramine).

medications of interest (Figure 1). The patient population from which the cohort was selected consisted of all primary care ambulatory veteran outpatients within VISN 11 aged 65 and older during the second and third quarters of 2004 $(\mathrm{n}=85,725)$. To be included in the cohort, patients had to be receiving a prescription for one or more of the five highrisk medications $(n=2,753)$. Each prescriber who ordered one of the high-risk medications for patients in this cohort received the first-stage intervention. For the second-stage intervention, a subgroup of patients in the cohort was identified. This subgroup included any patient in the cohort who had a scheduled appointment at a VA ambulatory care setting during the first 4 months of $2005(n=801)$. Each prescriber who was scheduled to see one or more patients in the subgroup received the second stage of the intervention.

\section{Intervention}

Selection of the targeted medications began with an examination of prescription volume for all Beers criteria medications. Five commonly prescribed drugs were targeted for the intervention and are operationally defined as "high risk" for purposes of this study: three tricyclic antidepressants (amitriptyline, imipramine, and doxepin) and two benzodiazepine sedative-hypnotics (chlordiazepoxide and diazepam). All of these agents are included in the VA formulary. These particular medications were selected based on several criteria: They were being prescribed in a significant portion of elderly patients in the VA population, they are categorized as potentially inappropriate drugs and are considered to have adverse outcomes of high severity according to Beers, a preponderance of evidence suggests that they should not be used in older patients, ${ }^{11,23,24}$ and one or more therapeutic alternatives with a better safety profile are readily available. There was consensus among the study investigators involved in the selection process (AM, SC, MS, PW, PR) that these five medications stood out from the rest of the Beers list by virtue of the clear-cut evidence 
documenting their toxicity in older patients and that primary care physicians would generally agree that these medications should be avoided in elderly people.

A two-stage intervention was implemented. The first stage of the intervention, introduced in October 2004, was a real-time medication-specific alert stating "not recommended for patients over the age of 65" (Figure 2). The alert appeared on the medication order screen of a patient's electronic record whenever a prescriber ordered one of the five high-risk drugs. The alert was provided in a generic information field that is routinely used for a variety of other drug-specific alerts. Once the warning had been activated in the electronic medical record, every prescriber who was ordering an outpatient prescription for one of the five highrisk medications would receive this message. This warning displayed to all clinicians writing for one of the high-risk agents, independent of patient-specific criteria such as age.

The second-stage intervention linked administrative patient appointment data with prescription data to target a group of prescribers. These prescribers were chosen, because they each had one or more upcoming appointments, during the first 4 months of 2005 , with one or more patients who were receiving one or more high-risk medications. These prescribers were specifically targeted, because they had the greatest opportunity to change their prescribing behaviors at a point in time close to the patient's office visit. Each targeted prescriber received a letter accompanied by a list of patients with upcoming appointments. At the end of each preceding month (i.e., in the final 10 days of December 2004 and January, February, and March 2005), a personally addressed letter (Appendix A) that all of the hospital chiefs of staff had reviewed, revised, and approved was sent, along with an outline of the project, to each targeted prescriber who was scheduled to see one or more patients during the following months. The letter was sent from the Chief Medical Officer of the VISN through the prescriber's Chief of Staff. The letter briefly described the rationale and goal of the project. It notified recipients that they had been targeted for the intervention, because they had written one or more prescriptions for a high-risk medication for one or more older patients who were scheduled to see them during the coming month. The letter asked the recipients to consider discontinuing or changing the medication and stated that the prescribing of high-risk medications would be tracked

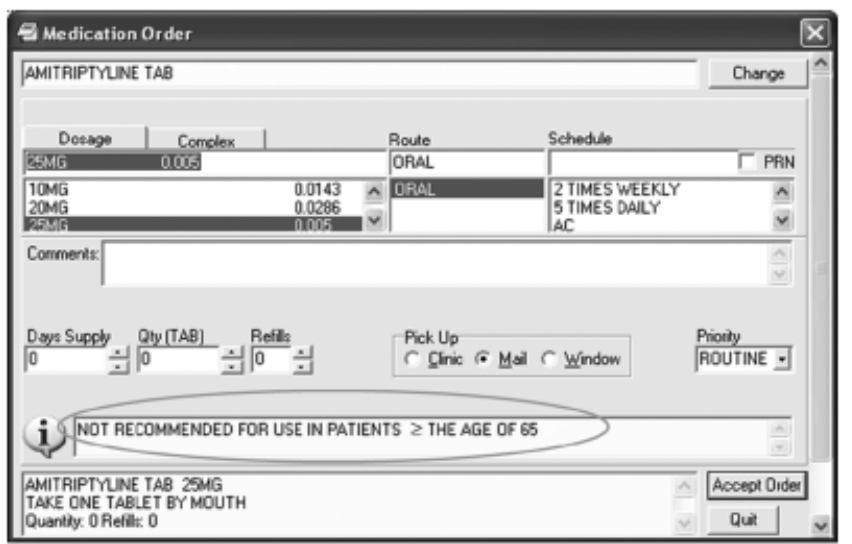

Figure 2. Stage 1 intervention: Drug-specific real-time alert. prospectively. There were no explicit incentives or penalties to compel change in the prescribers' behavior. There were three attachments to the letter: a copy of the most recent Beers criteria article, ${ }^{2}$ a list of suggested alternatives to each of the high-risk medications, and a list of older patients receiving one or more of the medications who had an appointment with the prescriber during the upcoming month. Because each targeted prescriber was identified at the beginning of each month of the intervention, prescribers could each receive up to four letters according to the number of months that they had appointments with patients during this intervention. Prescribers did not receive a letter if they did not prescribe any high-risk medications or did not have an appointment during the intervention period with a patient receiving a high-risk medication.

\section{Data Collection, Outcomes, and Analyses}

Preintervention data were collected before implementation of the Stage 1 intervention, during the second (April-June) and third (July-September) quarters of 2004 to identify characteristics of the cohort. Postintervention data were collected during the second and third quarters of 2005, after implementation of the Stage 1 and Stage 2 interventions. The primary outcome was the absence of a prescribed highrisk medication for each patient in the cohort during the postintervention period. The primary outcome focused on the entire cohort for two reasons; this approach provided a more conservative estimate of the effect of the intervention, and the intervention may have had a carryover effect onto prescribers that extended beyond the intervention period.

A secondary outcome examined the absence of a prescribed high-risk medication for each patient in the subgroup of the cohort. All electronic charts for the subgroup were reviewed to evaluate changes in any high-risk prescriptions. Using a standardized data abstraction form, two trained research assistants each reviewed half of the charts to ascertain whether the high-risk medication was continued, continued with a documented rationale for use, changed to an alternative medication, or discontinued during the clinic appointment. A study investigator $(\mathrm{BH})$ oversaw the chart review to ensure consistency between reviewers and answer questions.

Other secondary analyses included a comparison of changes in any high-risk medications for patients in the subgroup with changes for patients who were in the cohort but not part of the subgroup. This comparison was conducted to compare patients who were exposed to the Stage 1 and Stage 2 interventions (subgroup) with those who were exposed only to the Stage 1 intervention (remaining cohort). Additional analyses included the extent of prescribing changes for each of the five high-risk medications.

The McNemars chi-square test for paired samples compared changes in any high-risk medication from the postintervention period to the preintervention period. Separate analyses for changes in individual medications were also conducted using the McNemars test. All data were analyzed using SPSS version 14.0 (SPSS Inc., Chicago, IL).

\section{RESULTS}

There were 2,753 unique patients identified in the cohort; and 801 unique patients met inclusion criteria for the sub- 
group (those in the cohort who had at least one ambulatory care appointment during the first 4 months of 2005) and were available for follow-up chart review. There were 264 prescribers responsible for the high-risk prescriptions for patients in the subgroup. These prescribers received an average of 2.2 letters during the second-stage intervention period, for a total of 569 letters.

General characteristics of the identified cohort are listed in Table 1. The cohort was predominantly older white men. More than $70 \%$ of the cohort had hypertension, diabetes mellitus, or both. More than half of the patients in the cohort had heart disease.

In the entire cohort, $50.7 \%$ of patients had the highrisk medications discontinued, resulting in a significant decrease in the number of patients who were prescribed highrisk medications from the preintervention period to the postintervention period $(P<.001)$. Of the 801 patients in the subgroup, $72.0 \%(\mathrm{n}=577)$ had one or more high-risk medications discontinued $(P<.001)$. In contrast, of the remaining 1,952 patients whose prescribers received only the first intervention (i.e., real-time flag), $42.0 \%$ had high-risk medications discontinued $(P<.001)$.

Within the subgroup, high-risk medications were discontinued without replacement medication in $41.5 \%$ $(\mathrm{n}=332)$ of patients and discontinued with a change to an alternative agent in $30.6 \%(n=245)$ of patients. Highrisk medications were continued with documentation in $8.0 \%(n=64)$ of patients. The most common reasons for continuation of a high-risk medication were "patient is stable on the medications and does not wish to change," "patient was evaluated at this visit, and the provider feels that the drug is appropriate at this time," "patient denies any harmful side effects," "the medication is working well," "the patient does not want to try a new drug," and "a new

\begin{tabular}{lr}
\hline \multicolumn{1}{c}{ Table 1. Characteristics of the Cohort $(\mathbf{N}=\mathbf{2 , 7 5 3 )}$} \\
\hline \multicolumn{1}{c}{ Characteristic } & \multicolumn{1}{c}{ Value } \\
\hline Age, mean $\pm \mathrm{SD}^{*}$ & $72.5 \pm 5.9$ \\
Male, $\mathrm{n}(\%)$ & $2,683(95.1)$ \\
Race, $\mathrm{n}(\%)$ & \\
White & $1,533(55.7)$ \\
Black & $126(4.6)$ \\
Hispanic & $7(0.3)$ \\
American Indian or Alaska Native & $1(0.04)$ \\
Missing or unknown & $1,086(39.5)$ \\
Comorbid conditions, $\mathrm{n}(\%)^{\dagger}$ & \\
Hypertension & $2,357(85.6)$ \\
Diabetes mellitus & $2,000(72.6)$ \\
Ischemic heart disease & $1,645(59.8)$ \\
Depression & $653(23.7)$ \\
\hline Heart failure & $391(14.2)$ \\
Number of high-risk medications, mean $\pm \mathrm{SD}^{*}$ & $1.02 \pm 0.15$ \\
\hline
\end{tabular}

* Age as of the preintervention period (second and third quarters of 2004).

${ }^{\dagger}$ Comorbid conditions based on administrative International Classification of Diseases, Ninth Revision, codes. Patients may have had more than one of the comorbidities listed and, in addition, may have had comorbidities other than the five abstracted for this study.

$\mathrm{SD}=$ standard deviation. drug may cause more problems." In some instances, the clinician expressed a concern about stopping the drug too quickly and indicated that the drug dosage would be gradually decreased over time (e.g., "veteran is amenable to reducing doxepin from $50 \mathrm{mg}$ to $25 \mathrm{mg}$ and will consider further discontinuation though reluctant given the number of years of use. No dry mouth, constipation, or other side effects reported." Another example of reducing the dosage was, "the patient has been on chlordioazepoxide for a long time. The patient said he tried to go off Librium and he had severe withdrawal effects but he is willing to taper off."

Table 2 shows the extent of changes in prescribing according to medication. There was a significant decrease in the number of patients prescribed all five medications from the preintervention period to the postintervention period. In addition, the table shows results for the medications according to the level of intervention. In the group that received only the first stage of the intervention, the range of patients who were no longer prescribed high-risk medications was $30.2 \%$ to $48.0 \%$. In the group that received the first and second stages of the intervention, the range of patients no longer prescribed high-risk medication was $50.0 \%$ to $78.4 \%$. More than half of all opportunities $(57 \%)$ for changing high-risk medications were related to amitriptyline. An additional $20 \%$ of all opportunities were related to diazepam, $10 \%$ to doxepin, $7 \%$ to imipramine, and $6 \%$ to chlordiazepoxide.

To assist in understanding the effect of the staged intervention, the number of unique patients, aged 65 and older receiving one or more of the five high-risk medications during the year before implementation of the intervention and during the year after completion of the intervention was determined. On average, in the four quarters before the Stage 1 intervention, there were 2,432 older patients who received at least one of the five high-risk medications. In the year after completion of the Stage 2 intervention, the average number of patients who received at least one of the five medications was 1,485: a 39\% decrease. The Stage 1 intervention (real-time flag) continued to remain active in the year after completion of the Stage 2 intervention, although the absolute the number of unique patients who received one or more high-risk medications remained fairly constant during each quarter of that year (range 1,4571,501 patients).

\section{DISCUSSION}

This article reports on an effective intervention using a combination of targeted education and administrative oversight in reducing the number of older veterans in an outpatient setting prescribed high-risk medications. This result, when applied broadly, could have significant cost and quality implications in the medical care of elderly people.

Various interventions have been developed and tested to reduce inappropriate medication use and improve evidencebased prescribing. Strategies to alter prescribing practices include academic detailing, broad educational outreach, and computerized decision support. ${ }^{13,14,17-19,22,25,26} \mathrm{An}$ interrupted time series evaluation found that a drug-specific alert reduced the percentage of patients receiving high-risk medications $35 \%$ after controlling for baseline trend. ${ }^{18}$ In 
Table 2. Number of Unique Patients with a Prescription for One or More High-Risk Medications

\begin{tabular}{|c|c|c|c|}
\hline & Preintervention & Postintervention & \\
\hline Medication & & & Change, \%* \\
\hline Entire cohort (any high-risk medication) ${ }^{\dagger}$ & 2,753 & 1,357 & -50.7 \\
\hline Amitriptyline & 1,608 & 729 & -54.7 \\
\hline Chlordiazepoxide & 160 & 86 & -46.3 \\
\hline Diazepam & 556 & 303 & -45.5 \\
\hline Doxepin & 288 & 160 & -44.5 \\
\hline Imipramine & 208 & 113 & -45.7 \\
\hline Stage 1 intervention only (any high-risk medication) & 1,952 & 1,133 & -42.0 \\
\hline Amitriptyline & 1,149 & 598 & -48.0 \\
\hline Chlordiazepoxide & 109 & 75 & -31.2 \\
\hline Diazepam & 377 & 263 & -30.2 \\
\hline Doxepin & 210 & 137 & -34.8 \\
\hline Imipramine & 168 & 93 & -44.6 \\
\hline Stage 1 and 2 intervention (any high-risk medication) & 801 & 224 & -72.0 \\
\hline Amitriptyline & 459 & 132 & -71.2 \\
\hline Chlordiazepoxide & 51 & 11 & -78.4 \\
\hline Diazepam & 179 & 40 & -77.7 \\
\hline Doxepin & 78 & 23 & -70.5 \\
\hline Imipramine & 40 & 20 & -50.0 \\
\hline
\end{tabular}

* All pre-post comparisons were significant at $P<.001$.

${ }^{\dagger}$ Prescribers in the entire cohort received a combination of the Stage 1 intervention only or the Stage 1 intervention plus the Stage 2 intervention. Any high-risk medication refers to the medications listed in the table.

the current study, the cohort that was exposed to the Stage 1 intervention (real-time computer alert) but not the Stage 2 intervention had a $45 \%$ reduction in prescriptions for highrisk medications. In the previous study, the medications included the same five examined in the current study plus additional high-risk medications. ${ }^{18}$

Although the results from these two studies are similar, there are several important things to consider. First, the context and design of the computer alerts can influence the findings. In both studies, the electronic alert was "drug specific" not "patient specific." As a result, the alert was displayed every time a prescriber ordered one of the highrisk medications. Patient-specific variables such as age were not a factor in the display of the alert. This type of drugspecific alert could increase prescriber burden and decrease prescriber vigilance. A more-effective alert might incorporate drug- and patient-specific variables. Also, the design of the alert varied in the two studies. In the previous study, the drug alert included alternative medications on screen. ${ }^{18}$ With one step, the prescriber could order the alternative medications, and to continue with the order for a high-risk medication, the prescriber was required to perform an additional step, whereas in the current study, the alert was "passive" and did not provide on-screen alternative medications. Additional steps were required to order the alternative medications rather than continuing the order for the high-risk medication. Given these differences in design, it would be reasonable to expect that the alert in the previous study would be more effective. The differences in results between the two studies may be due to the number of medications targeted, differences in the hospital systems (e.g., VA vs non-VA clinics), and differences in the structure and function of the electronic health records.
The Stage 2 intervention in this study offered several unique aspects that seemed to successfully change prescriber behavior. First, using administrative data, a targeted group of prescribers was identified to receive a personally relevant educational letter. By targeting specific prescribers, this intervention focused on those clinicians with the greatest opportunity to change prescribing behavior (e.g., a prescriber with no recent history of prescribing high-risk medications would be less likely to be affected), thereby reducing resource requirements and increasing the benefit:cost ratio of the intervention.

Second, along with the letter, the targeted group received a list of patients for whom the prescriber would soon have a convenient opportunity to affect with the new knowledge. Prescriber learning often occurs from consultations with colleagues who provide knowledge that can be directly applied to specific patients during the office visit. ${ }^{27-29}$ Therefore, the intervention in this study was designed to educate prescribers at a point in time close to the patient's office visit. Clinicians could apply the knowledge gained from the intervention to relevant patients who were scheduled to be seen in the upcoming month. In addition, the education may carry over to affect other patients seen by the prescriber at a later date.

Third, the intervention was focused on a limited number of specific medications from the Beers list. There was consensus among the study physicians that these five medications stood out from the rest of the Beers list by virtue of the clear-cut evidence documenting their toxicity in older patients and the availability of less-harmful alternative medications. Including only these five medications limited the scope of the intervention and also avoided questions that may have arisen regarding some medications on the 
Beers list for which controversy exists or where exceptions for use of the medication are more common.

Finally, the letter was generated and signed with administrative support from colleagues. To be most effective, prescriber education should be conducted by someone viewed as a colleague or opinion leader. ${ }^{30}$ In this study, the educational letter was generated after several revisions from the Medical Chiefs of Staff and sent by the VISN Chief Medical Officer (CMO). In general the VISN CMO and the Chief of Staff are physician colleagues who other clinicians may view as opinion leaders. In addition, there is the possibility that the information, coming from the $\mathrm{CMO}$ of the healthcare system, along with the knowledge that future prescribing of these agents would be tracked, may also carry some element of performance expectation.

Although the results of this quality improvement project are intriguing, there are several limitations. First, there was no control group identified for this project, because it was designed for quality improvement rather than a rigorous test of an intervention. Therefore, the study cannot account for changes that would occur because of regression to the mean, temporal trends, or other factors occurring during the intervention period. It is possible that similar reductions in the number of patients prescribed high-risk medications occurred despite this intervention, although the magnitude of the reduction suggests otherwise. In addition, the number of unique patients who were receiving one or more high-risk medications was relatively flat during the year before the intervention. Nevertheless, future studies should include a control group to provide a moreaccurate estimate of the effect. Also, the intervention was twofold, and the second stage contained several components (two educational pieces, a request by an authority figure to change prescribing, and patient information to facilitate compliance with the request). The study design was not able to determine which part or parts of the interventions were more successful, although it appears that the components of the second-stage intervention may have been more successful given that much-larger decreases in the number of patients prescribed high-risk medications was seen in the subgroup that received both stages of the intervention than in the subgroup that received only the first stage of the intervention. In addition, the number of unique patients who were receiving one or more high-risk medications was relatively flat during the year after completion of the second-stage intervention while the first stage intervention continued. Although the interventions were targeted at prescribers, no data were collected on prescriber characteristics. Ideally, these data would provide information useful for predicting response to other interventions. Future studies should include data on prescribers to allow targeting in future interventions. Finally, this study used a combination of healthcare information technology and infrastructure within a regional VA system. Although the intervention may be replicable at other VA systems, it may be less easily generalized to other non-VA healthcare systems that lack the ability to link outpatient medications, prescriber identities, and outpatient appointments. Nevertheless, as healthcare systems increase their information technology resources, approaches such as this one, linking administrative and clinical data sources, will become increasingly feasible.
The multimethod intervention in this study reduced the prescribing of high-risk medications to older veterans by $50 \%$. These findings should be confirmed in future studies using a control group and in non-VA settings. If effective, this intervention could have significant quality and cost implications in the medical care of older adults.

\section{ACKNOWLEDGMENTS}

Conflict of Interest: The editor in chief has reviewed the conflict of interest checklist provided by the authors and has determined that the authors have no financial or any other kind of personal conflicts with this manuscript. This project was supported by the Veterans Integrated Service Network 11.

Author Contributions: Study concept and design $(\mathrm{BH}$, SC, AM, KS, MS, PW, PR). Development of intervention materials (BH, TM, KS, PR). Data collection (AZ, BH, TM). Analysis and interpretation of data (AZ, BH, TM, SC, AM, KS, MS). Preparation of manuscript (AZ, KS, BH, TE, $\mathrm{SC}, \mathrm{AM})$. Critical review of manuscript (BH, SC, AM, KS, MS, PW).

Sponsor's Role: The sponsor had no role in the methods, analysis, interpretation of the data, the preparation of the manuscript, or the decision to submit for publication.

\section{REFERENCES}

1. Committee on Quality of Heath Care in America. To Err Is Human: Building a Safer Health System. Institute of Medicine, 2000. National Academy Press, Washington, DC.

2. Zhan C, Sangl J, Bierman AS et al. Potentially inappropriate medication use in the community-dwelling elderly: Findings from the 1996 Medical Expenditure Panel Survey. JAMA 2001;286:2823-2829.

3. Goulding MR. Inappropriate medication prescribing for elderly ambulatory care patients. Arch Intern Med 2004;164:305-312.

4. Gurwitz JH, Rochon P. Improving the quality of medication use in elderly patients: A not-so-simple prescription. Arch Intern Med 2002;162: 1670-1672.

5. Hanlon JT, Schmader KE, Boult C et al. Use of inappropriate prescription drugs by older people. J Am Geriatr Soc 2002;50:26-34.

6. Curtis LH, Ostbye T, Sendersky V et al. Inappropriate prescribing for elderly Americans in a large outpatient population. Arch Intern Med 2004;164: 1621-1625.

7. Simon SR, Chan KA, Soumerai SB et al. Potentially inappropriate medication use by elderly persons in U.S. Health Maintenance Organizations, 2000-2001. J Am Geriatr Soc 2005;53:227-232.

8. Steinman MA, Rosenthal GE, Landefeld CS et al. Conflicts and concordance between measures of medication prescribing quality. Med Care 2007;45: 95-99.

9. Beers MH. Explicit criteria for determining potentially inappropriate medication use by the elderly. An update. Arch Intern Med 1997;157:1531-1536.

10. Beers MH, Ouslander JG, Rollingher I et al. Explicit criteria for determining inappropriate medication use in nursing home residents. UCLA Division of Geriatric Medicine. Arch Intern Med 1991;151:1825-1832.

11. Fick DM, Cooper JW, Wade WE et al. Updating the Beers criteria for potentially inappropriate medication use in older adults: Results of a U.S. consensus panel of experts. Arch Intern Med 2003;163:2716-2724.

12. Pugh MJ, Fincke BG, Bierman AS et al. Potentially inappropriate prescribing in elderly veterans: Are we using the wrong drug, wrong dose, or wrong duration? J Am Geriatr Soc 2005;53:1282-1289.

13. Bero LA, Grilli R, Grimshaw JM et al. Closing the gap between research and practice: An overview of systematic reviews of interventions to promote the implementation of research findings. The Cochrane Effective Practice and Organization of Care Review Group. BMJ 1998;317:465-468.

14. Davis DA, Thomson MA, Oxman AD et al. Evidence for the effectiveness of CME: A review of 50 randomized controlled trials. JAMA 1992;268:1111-1117.

15. Fick DM, Maclean JR, Rodriguez NA et al. A randomized study to decrease the use of potentially inappropriate medications among community-dwelling older adults in a southeastern managed care organization. Am J Manage Care 2004;10:761-768. 
16. Monane M, Matthias DM, Nagle BA et al. Improving prescribing patterns for the elderly through an online drug utilization review intervention: A system linking the physician, pharmacist, and computer. JAMA 1998;280: 1249-1252.

17. Raebel MA, Charles J, Dugan J et al. Randomized trial to improve prescribing safety in ambulatory elderly patients. J Am Geriatr Soc 2007;55:977-985.

18. Smith DH, Perrin N, Feldstein A et al. The impact of prescribing safety alerts for elderly persons in an electronic medical record: An interrupted time series evaluation. Arch Intern Med 2006;166:1098-1104.

19. Soumerai SB, Avorn J. Principles of educational outreach ('academic detailing') to improve clinical decision making. JAMA 1990;263:549-556.

20. Spinewine A, Swine C, Dhillon S et al. Effect of a collaborative approach on the quality of prescribing for geriatric inpatients: A randomized, controlled trial. J Am Geriatr Soc 2007;55:658-665.

21. Van Eijk ME, Avorn J, Porsius AJ et al. Reducing prescribing of highly anticholinergic antidepressants for elderly people: Randomised trial of group versus individual academic detailing. BMJ 2001;322:654-657.

22. Greco PJ, Eisenberg JM. Changing physicians' practices. N Engl J Med 1993;329:1271-1273.

23. Fu AZ, Liu GG, Christensen DB. Inappropriate medication use and health outcomes in the elderly. J Am Geriatr Soc 2004;52:1934-1939.

24. Meredith S, Feldman PH, Frey D et al. Possible medication errors in home healthcare patients. J Am Geriatr Soc 2001;49:719-724.

25. Baran RW, DuChane J, Parker L et al. Effectiveness of academic detailing in the managed care environment: Improving prescribing of lipid-lowering agents. J Manage Care Pharm 1996;2:148-157.

26. O'Connell DL, Henry D, Tomlins R. Randomised controlled trial of effect of feedback on general practitioners' prescribing in Australia. BMJ 1999;318: 507-511.

27. Elliott TE, Elliott BA. Physician acquisition of cancer pain management knowledge. J Pain Symptom Manage 1991;6:224-229.

28. Elliott TE, Murray DM, Oken MM et al. Improving cancer pain management in communities: Main results from a randomized controlled trial. J Pain Symptom Manage 1997;13:191-203.

29. Knowles MS. Self-Directed Learning: A Guide for Learners and Teachers. New York, NY: The Adult Education Company, 1975.

30. Soumerai SB, McLaughlin TJ, Avorn J. Quality assurance for drug prescribing. Qual Assur Health Care 1990;2:37-58.

\section{Appendix A}

\section{Department of
Veterans Affairs}

\section{Memorandum}

Date: « Date 》

From: Chief Medical Officer, VISN 11 (10N11)

Subj: Reduction in use of "Potentially Inappropriate Medications" in outpatient veterans over age 65 in VISN 11.

To: $\ll$ Name of Provider $\gg$

THRU: Chief of Staff $(11) \ll$ signature 》

1. As part of the ongoing effort to continuously improve the quality of care of veterans in VISN 11, steps are underway to reduce or even to eliminate the use of certain pharmacological agents that have been shown in patients over age 65 to have less or equivalent efficacy yet a less favorable side- effect profile, compared to other agents. This memorandum urges your support in this effort.

2. Attached for your consideration is a review article on "Potentially Inappropriate Medications for use in the Elderly" from Archives of Internal Medicine. My office, in collaboration with the VISN 11 Geriatrics \& Extended Care Service Line, is initially focusing on reducing or eliminating the use in veterans over age 65 years of only these five agents identified in that article:

\section{- Amitriptyline}

- Diazepam

- Doxepin

- Imipramine

- Chlordiazepoxide

3. Pharmacy records indicate that you wrote one or more prescriptions for one or more of these agents during the third and fourth quarters of FY04. To support your efforts to limit the use of these agents in older patients in the future, attached is a list of over-age-65 patient(s) for whom you prescribed one or more of the preceding agents; and who are appointed to see you during January 2005. You are encouraged to select an alternative agent for these patients at the times of their next appointments (a third attachment to this letter identifies those agents suggested as preferable alternatives by the VISN's Pharmacy Benefits Manager). You are also requested to not write new prescriptions for any of these agents to any veteran over age 65 .

4. To further assist this process, as of January 1 prescription orders in CPRS for these agents will receive a warning that they are "potentially inappropriate for patients over age 65 years."

5. You will receive a memorandum similar to this, accompanied by a list of patients already receiving these agents who are scheduled to see you the following month, each month through May (excepting any month in which no such patients are scheduled to see you). Prescribing patterns within the medical center, and in VISN 11, will be assessed at that time relative to FY04. It is our hope and expectation that prescriptions of these agents to veterans over age 65 will show a significant decline. If this is not the case, alternative approaches to achieving the desired end will be considered.

6. The Institute of Medicine has estimated that adverse drug reactions cost over $\$ 100$ billion annually in the U.S. You are being asked to do your part to curb those adverse effects in a small but particularly vulnerable subgroup of your clinical population. The success of the approach is dependent on your participation as described. Thank you in advance for your cooperation.

Pamela Reeves, MD 